\section{Achieving Zero Catheter-Associated Urinary Tract Infections in a Neurosurgery Intensive Care Unit}

To the Editor-The use of urinary catheters to accurately monitor urine output is always indicated in critically ill patients. Especially in the neurosurgery intensive care unit (ICU), the urinary catheter is frequently used for urinary retention or incontinence. However, the complications of catheter-associated urinary tract infection (CAUTI) following the insertion of catheters can cause significant morbidity. To improve the quality of care, several strategies were developed for prevention of CAUTI. ${ }^{1-4}$ These strategies included (1) reducing the unnecessary use of urinary catheters, (2) using aseptic procedures during catheter insertion, (3) insertion of catheters by trained personnel, (4) aseptically maintaining the catheter, and (5) early removal of catheter. ${ }^{2-4}$ However, studies that investigate the impact of this care bundle for prevention of CAUTI in neurosurgery ICUs is scarce. Since July 2012, 1 quality improvement project to reduce CAUTI was implemented in our neurosurgery ICU. This study was conducted to evaluate the effect of the implementation of 1 CAUTI care bundle in the neurosurgery ICU.

This study was carried out in a neurosurgery ICU at a regional teaching hospital with 10 adult ICU beds and 1 intensivist. The insertions of urinary catheters for male patients were performed by nurse practitioners, and critical care nurses were responsible for insertion of urinary catheters for female patients. Since July 2013, the CAUTI care bundle was implemented in the entire ICU. The bundle includes several components, including hand hygiene, ensuring that there are the indications for urinary catheter insertion, use of aseptic technique by trained healthcare providers, maintenance of a sterile closed drainage system, keeping the drainage bag below the level of bladder, daily review of indications for the urinary catheter, early removal of unnecessary catheters, and avoiding routine changing of catheters or drainage bags.

CAUTI was defined according to Centers for Disease Control and Prevention guidelines. ${ }^{5}$ Outcomes including CAUTI per 1,000 catheter-days, CAUTI per 1,000 inpatient-days, and catheter utilization rates (days of catheter use divided by total inpatient-days) were measured. To evaluate the impact of bundle care on the rate of CAUTI over time, we divided the study time into 4 parts, including the preintervention period (January 2012-June 2012), postintervention period 1 (July 2012-December 2012), postintervention period 2 (January 2013-June 2013), and postintervention period 3 (July 2013December 2013). The $\chi^{2}$ for trend was used to assess temporal changes in incidence. A $P<.05$ was considered to be statistically significant.

During the 2-year period, there were a total of 16 episodes of CAUTI, and the catheter utilization rate was 0.83 . The rate of CAUTI was 2.13 per 1,000 inpatient-days and 2.55 per 1,000 catheter-days. The rate of CAUTI significantly declined from 3.86 per 1,000 catheter-days in the preintervention period to 0 per 1,000 catheter-days in postintervention period $3(P=.026$; Figure 1$)$. In addition, the rate of CAUTI per 1,000 inpatient-days showed a similar trend that significantly decreased from 3.01 per 1,000 inpatient-days in the preintervention period to 0 per 1,000 inpatient-days $(P=.037$; Figure 1). In contrast, the catheter utilization rate significantly increased from 0.78 in the preintervention period to 0.85 in postintervention period $3(P<.0001)$.

In this 2-year study, we demonstrated that zero CAUTI can be achieved in the neurosurgery ICU. It is consistent with a previous study ${ }^{6}$ in a district hospital in Kenya that showed that zero CAUTI was achieved and maintained for 7 weeks

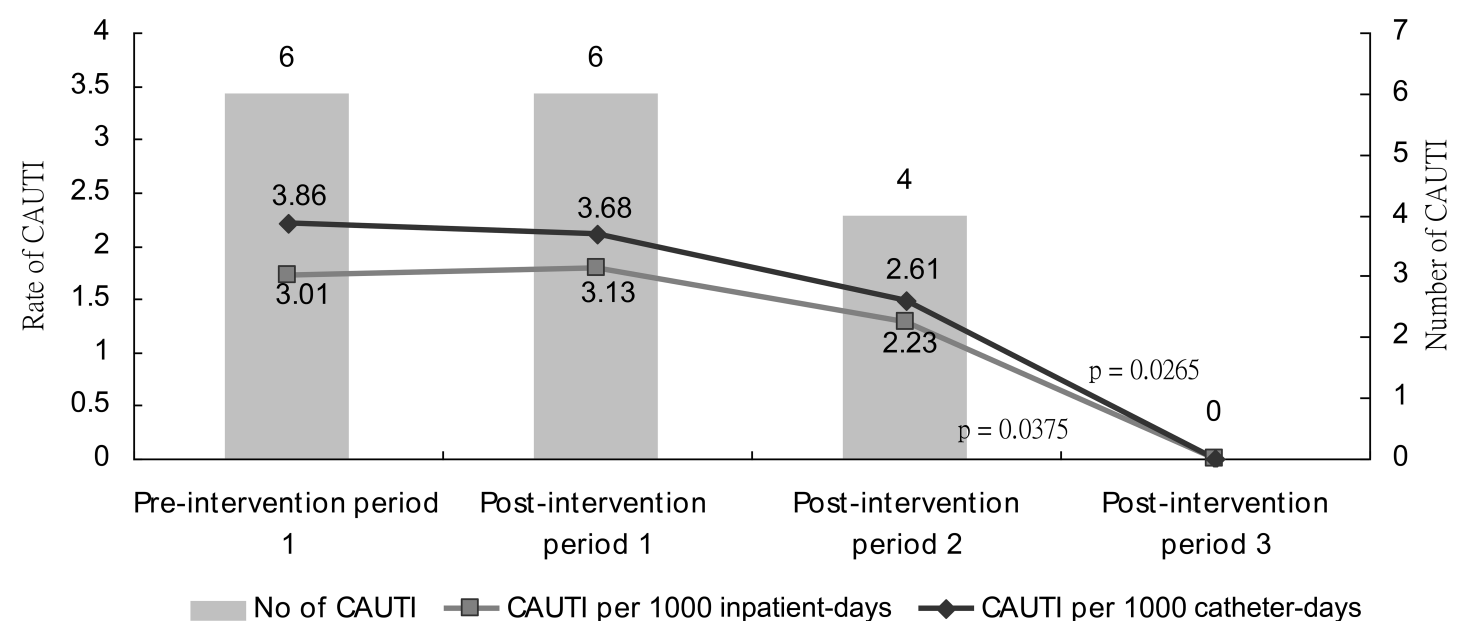

FIGURE 1. Rate and number of catheter-associated urinary tract infections (CAUTIs) in pre- and postintervention periods. 
after a multifaceted intervention in the medical ward. All of these encouraging findings indicate that CAUTI can be preventable by a multidisciplinary team care bundle.

In contrast to previous reports ${ }^{6}$ that the catheter utilization ratios significantly decreased from 0.14 to $0.09(P<.001)$, the catheter utilization ratio remained high and increased from 0.78 to $0.85(P<.001)$. This large difference in utilization ratio reflects different study settings, for example, neurosurgery ICU versus medical ward. Most of the patients in this study were unconscious and needed close monitoring of urine output in the neurosurgery ICU; therefore, the catheter utilization ratio could be higher than in other settings. However, the intensivists should still adhere to the bundle, for example, daily review of the indications urinary catheters and early removal of the catheter to avoid unnecessary use of urinary catheters.

In conclusion, the rate of CAUTI in the neurosurgery ICU can be reduced to zero after implementation of a prevention care bundle in spite of a high catheter utilization ratio.

\section{ACKNOWLEDGMENTS}

Potential conflicts of interest. All authors report no conflicts of interest relevant to this article. All authors submitted the ICMJE Form for Disclosure of Potential Conflicts of Interest, and the conflicts that the editors consider relevant to this article are disclosed here. Wei-Ya Cheng, RN; ${ }^{1}$ Hsin-Lan Lin, $\mathrm{RN} ;{ }^{1}$ Yu-Hsiu Lin; ${ }^{2}$
Chih-Cheng Lai, MD; ${ }^{3}$ Chien-Ming Chao, $\mathrm{MD}^{3,4}$

Affiliations: 1. Department of Nursing, Chi Mei Medical Center, Liouying, Tainan, Taiwan; 2. Committee of Infection Control, Chi Mei Medical Center, Liouying, Tainan, Taiwan; 3. Department of Intensive Care Medicine, Chi Mei Medical Center, Liouying, Tainan, Taiwan; 4. Department of Nursing, Min-Hwei College of Health Care Management, Tainan, Taiwan.

Address correspondence to Chien-Ming Chao, Department of Intensive Care Medicine, Chi-Mei Medical Center, Liouying, Tainan, Taiwan (ccm870958@yahoo.com.tw).

Infect Control Hosp Epidemiol 2014;35(6):746-747

(C) 2014 by The Society for Healthcare Epidemiology of America. All rights reserved. 0899-823X/2014/3506-0023\$15.00. DOI: 10.1086/676440

\section{REFERENCES}

1. Marra AR, Camargo TZS, Goncalves P, et al. Preventing catheterassociated urinary tract infection in the zero-tolerance era. Am J Infect Control 2011;39:817-822.

2. Ducel G, Fabry J, Nicolle L, eds. Prevention of Hospital-Acquired Infections: A Practical Guide. 2nd ed. Geneva: World Health Organization, 2002.

3. Gould CV, Umscheid CA, Agarwal RK, Kuntz G, Pegues DA. Guideline for prevention of catheter-associated urinary tract infections 2009. Infect Control Hosp Epidemiol 2010;31:319-326.

4. Lo E, Nicolle I, Classen D, et al. Strategies to prevent catheterassociated urinary tract infections in acute care hospitals. Infect Control Hosp Epidemiol 2008;29(supp 1):S41-S50.
5. Centers for Disease Control and Prevention (CDC). Device-Associated Module: CAUTI. Atlanta: CDC, 2011.

6. Tillekeratne LG, Linkin DR, Obino M, et al. A multifaceted intervention to reduce rates of catheter-associated urinary tract infections in a resource-limited setting. Am J Infect Control 2014; 42:12-16.

\section{Bacteriostatic Effect of Mixtures of $1 \%$ Propofol with $4 \%$ Lidocaine versus $4 \%$ Lidocaine Alone: Regards on Microbiologic Studies in the Field of Anesthesiology}

To the Editor-Since many decades ago, we have seen the great incursion that infectious diseases have had in the field of anesthesiology: first, because of the emerging knowledge and advanced techniques related to the clinical management of medical conditions, and second, because of the importance of prevention of nosocomial infections when aseptic techniques are appropriately enforced in anesthetic practice.

Among 20 or more original articles based on the microbiology approach on anesthesiologic topics such as the bacteriostatic and probiotic properties of anesthetics, we want to mention 1 particular study by Sakuragi et $\mathrm{al}^{1}$ published in 1999 with the purpose of specifying the advantages, disadvantages, and areas for improvement in this line of investigation. In an experimental study with a frequent nosocomial pathogen (Escherichia coli), Sakuragi et al $^{1}$ mentioned the purpose of verifying the concentration-dependent antibacterial activity of lidocaine alone and when it is combined with propofol emulsion to compare this interaction, and they demonstrated the bacteriostatic effect of lidocaine under different concentration mixtures in a $1 \%$ propofol solution. They concluded that the addition of lidocaine, even at low concentrations, to propofol solution might be an innovative technique to decrease the hazard of nosocomial infections associated with bacterial extrinsic contamination of propofol. Methodologically, the colony count carried out by the authors through in vitro cultures is the gold standard to identify bacterial growth; however, in these cases, the use of new techniques is recommended, such as flow cytometry, which estimates susceptibility by means of bacterial viability outcomes. ${ }^{3}$ In the study by Pina-Vaz et al, ${ }^{4}$ the bacteriostatic effect of lidocaine was measured in terms of minimal inhibitory concentration following the flow cytometry technique; interestingly, this technique allowed for clarification of the mechanism of action through the different fluorescent stains and laboratory conditions. ${ }^{5}$

In the first observations made by Sakuragi et $\mathrm{al}^{1}$ about bacterial growth on propofol alone, we might say that there were coherent results, according to several case reports of outbreak infections associated with propofol contamination. ${ }^{6}$ 